\title{
Overview of current and future research and clinical directions for drug discontinuation: psychological, traditional and professional obstacles to deprescribing
}

\author{
Doron Garfinkel, ${ }^{1,2}$ IGRIMUP
}

\begin{abstract}
${ }^{1}$ Wolfson Medical Center, Holon, Israel

${ }^{2}$ Homecare Service, Israel Cancer Association, Givatayim, Israel
\end{abstract}

Correspondence to Professor Doron Garfinkel, Wolfson Medical Center, 55 Ben-Gurion Road, Bat Yam, 5932210, Israel; dorong@netvision.net.il

Received 23 April 2016 Revised 23 June 2016 Accepted 28 June 2016

EAHP Statement 6: Education and Research

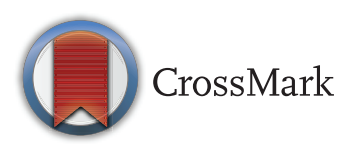

To cite: Garfinkel D IGRIMUP. Eur J Hosp Pharm 2017;24:16-20.

\section{ABSTRACT}

The vicious circle of age-related diseases, many experts and guidelines/drugs fuels the 21st century iatrogenic epidemic of inappropriate medication use and polypharmacy. There are no evidence-based medicine (EBM) 'guidelines' for treating older people, and knowledge gaps regarding dosage requirements. For all drugs, the positive benefit/risk ratio is decreasing/ inverted in correlation to very old age, comorbidity, dementia, frailty and limited life-expectancy (VOCODFLEX). Main obstacles to routine deprescribing are emotional/psychological myths; patient-doctor interactions are expected to be transformed into prescription; doctors are perceived as expert prescribers who wisely choose the right medication/s to treat all diseases. Although most 'guidelines' were not proven in older people, particularly VOCODFLEX, doctors are afraid of lawsuits and of the patient/family reaction if they do not follow all experts' recommendations. Doctors are frustrated facing uncertainty regarding the effectiveness of strategies to reduce polypharmacy and the lack of EBM indicating when to de-prescribe. When explicit criteria and 'drugs to avoid' are used alone, we may disregard undiagnosed harms imposed by the remaining drug groups and interactions. The best approaches are implicit tools that take into consideration EBM data, clinical circumstances and medical judgement. The Garfinkel Good Palliative-Geriatric Practice method recommends deprescribing of as many drugs as possible simultaneously, giving high priority to patient/family preferences. It was proven highly effective and safe in nursing departments and in community-dwelling elders, having significant economic benefits as well.

\section{INTRODUCTION}

Improved medical technology has resulted in a huge increase in lifespan in general, and in patients with age-related chronic, life-shortening diseases in particular. Nowadays, most adults experience timerelated increases in the number of incurable comorbidities, disability and suffering for increasingly prolonged periods before death. ${ }^{1}{ }^{2}$ This brought about a rapid increase in subpopulations of patients at risk of inappropriate medicines use and polypharmacy including the Very Old, those with CO-morbid, Dementia, Frailty (disability) Limited life-EXpectancy (also known as the VOCODFLEX group). ${ }^{1}{ }^{3}$ An increasingly growing imbalance is created between 'these patient groups' and younger healthy-supportive subpopulations that carry the unprecedented burden of medical, economic and social age-related problems referred to as 'the geriatric boom burden', and 'the Tsunami in 21st century health care. ${ }^{3}$

Traditional 20th century research, educational and clinical principles combine to create a leading 21st century epidemic of inappropriate medication use (IMU) and polypharmacy. Being completely aware of the differences between the two terms, in this article, the author sometimes addresses the terms polypharmacy and IMU together, as IMU and polypharmacy (IMUP) or 'the problem'. Adopting this practice avoids useless nosological nuisance while enabling practical consensus to combat the problem.

Apart from specific adverse drug events (ADE), the increased rate of drug-disease and drug-drug interaction related to the problem is associated with diverse negative clinical outcomes. These include cognitive, mental and functional impairments, delirium, weight loss, malnutrition, falls, hip fractures, incontinence, hospitalisations, nursing home $(\mathrm{NH})$ placement, decrease in quality of life (QoL) and death, as well as non-adherence. IMU is also associated with negative economic outcomes, including needless evaluations for ADE-related 'new symptoms' resulting from 'the polypharmacy cascade'. ${ }^{4} 5$ The various negative clinical and economic outcomes and the tools already suggested to reduce the problem were recently reviewed by us ${ }^{1}$ and many others. Assessing them in depth is beyond the scope of this manuscript; they are addressed in other papers in this issue. I concentrate on trying to define the problem, identify barriers and enablers, present the results of the Good Palliative-Geriatric Practice (GPGP) method and finally, suggest a new perception/approach for significantly reducing this 21 st century major, iatrogenic epidemic.

\section{DEFINING THE PROBLEM-WHY IS THE IATROGENIC EPIDEMIC EMERGING NOW?}

Living longer is associated with increased portion of subpopulations suffering from age-related comorbidities, dementia and disability. The higher the number of diseases, the higher the number of specialists involved. Experts and family physicians (FP) recommend interventions based on randomised controlled trials (RCTs) that are essential for establishing evidence-based medicine (EBM) guidelines. However, RCTs are usually based on a 'single disease model', in much younger, healthier 
populations with life expectancy of several decades. Older people and certainly VOCODFLEX subpopulations are excluded from RCTs, and trials in older populations are even more non-representative. ${ }^{6-8}$ Therefore, we do not really have EBM guidelines for most medications in older people, particularly in VOCODFLEX.

There is an ongoing debate regarding the number of medications that should be regarded excessive, making their accumulating effect a disease in itself (polypharmacy). This lack of consensus contributes to the confusion regarding the precise definition. Polypharmacy was vaguely defined as 'the administration of more medications than are clinically indicated', but facing the lack of EBM in older people, who decides what is really 'clinically indicated'. IMU refers to medication use that 'has more potential risk for harming than potential benefit, is less effective than alternatives available...or does not agree with accepted medical standards'. ${ }^{9}$ Back to square one, because we have no 'accepted standards' in most older people. Gnjidic et $a l^{10}$ suggested that five drugs represent the cut-off for the definition of polypharmacy as older adults taking more medications have increased risk of mortality, disability, frailty and falls. Fifty per cent of Medicare beneficiaries receive more than five medications. ${ }^{11}$ In older adults with cancer, $84 \%$ were receiving $>5$ medications, and $43 \%$ were receiving $\geq 10$ medications. ${ }^{12}$ Again, in order to minimise confusion and enable practical interventions I suggest defining 'the problem' as IMUP.

Boyd et $a l^{13}$ warn that adhering to current guidelines in elders with multiple comorbidities leads to inappropriate judgement, create perverse incentives and diminish their quality of care.

A sober, rational analysis of our overall therapeutic drug approach in older people reveals annoying, alarming consequences. It may be concluded that for most medications the positive benefit/risk ratio is non-existing or reversed, in correlation to age and the extent of having VOCODFLEX characteristics. Paradoxically, on the other hand of the scale, the severity of IMU increases in correlation to exactly the same characteristics plus the number of drugs (polypharmacy). Furthermore, the problem is aggravated due to "prescription cascades' where symptoms resulting from ADE are perceived as new symptoms caused by 'new' underlying diseases. This creates another iatrogenic vicious circle of overdiagnosis, useless evaluations, hospitalisations, overtreatment and IMU. These vicious circles may be sustained by continuing diagnostics and external incentives. ${ }^{14-17}$ Other contributing factors are the 'defensive medicine' attitudes of automatically following orders adhering to whatever guidelines we do have. The final consequences are significant health risks and bad QoL for an increasing number of older people for an increased period of time before death.

Nevertheless, FP and specialists continue to prescribe their 'field-specific, guideline based medications' to all patients, regardless of age and patient's characteristics sometimes until death. Considering our basic ethical axiom of 'first do not harm' this iatrogenic epidemic is at best inappropriate, and at worst, harmful to the patient.

All these negative, irrational consequences of IMUP result from the common situation where different doctors are 'responsible' for different medications. FP and experts usually never interfere with 'field specific guidelines' determined by other experts. This problem is usually defined as 'diffusion of responsibility' - the sociopsychological phenomenon whereby a person is less likely to take responsibility for action or inaction when others are present. Others entitle it 'the bystander effect'; when different professionals are involved in treating a complex patient, chaos and inaction may result. Each caregiver may assume a passive role, expecting another MD to bear the burden of authority and responsibility. ${ }^{18}$ Eventually, a scheduled, formal drug re-evaluation is usually never performed in older people, neither in hospitals or $\mathrm{NH}$ nor in the community. In the author's experience, this unfortunate situation where basically patients do not have one, knowledgeable 'all drugs' case manager' leads to poor compliance, anxiety and confusion to patients, families and caregivers.

\section{CHOOSING THE BEST APPROACH TO COMBAT IMUP}

These are covered in other papers in this issue. They are classified as criteria-based explicit tools, which can be lists of potentially inappropriate medicines for use as part of medication review, for example Beers lists, STOPP/START, and so on, ${ }^{19-23}$ and implicit (judgement-based) tools.

We are not yet at the point where in multidrug situations, we are sure which drugs are causing which reactions. While some explicit tools, for example, STOPP/START, do take account of the patient's clinical situation, focus on potentially inappropriate medicines generally may lead clinicians who review medicines to assume that all other drug combinations continued are 'safe'. The author advocates a different approach that may be classified among the implicit tools. These approaches take into consideration both EBM data and clinical circumstances. ${ }^{24-28}$

A most significant predictor of IMU is the number of drugs (polypharmacy). Therefore, the best solution to prevent/cure the IMUP epidemic is deprescribing of as many drugs as possible simultaneously, with patient/family approval. ${ }^{1}{ }^{29}{ }^{30}$ Based on this hypothesis, the author has developed the GPGP algorithm and method that combines EBM with clinical judgement giving high priority to patient/family preferences. Rethinking/ re-evaluation is performed for each drug while discussing with the patient/family the pros and cons of each drug with the main goal being QoL. With their consent, as many 'non-life-saving' drugs as possible are discontinued simultaneously. In a situation where patients felt that the doctors were just giving up by reducing medications, gaining the patient/family trust is obviously of crucial importance to reach a mutual understanding of the benefit of the process.

The GPGP calls for a less aggressive approach in reaching rigid target goals (blood pressure, serum glucose and lipid concentrations). There is also an important, beneficial medicolegal byproduct-having the patient/family involved in establishing the therapeutic approach reduces doctor's fear of lawsuits. GPGP was the first method to prove that effective reduction in polypharmacy by simultaneous deprescribing of many medications resulted in significantly improved clinical outcomes, both in nursing departments ${ }^{29}$ and in community-dwelling elders. ${ }^{30}$ In disabled patients it was associated with a significant decrease in annual mortality, morbidity, referrals to acute care hospitals and cost reduction. In community-dwelling elders GPGP resulted in improved functional, mental and cognitive status with high level of patient/family satisfaction. There were no significant ADE to the substantial deprescribing. The GPGP was suggested as a basic perception for addressing IMUP. ${ }^{31} 32$

\section{BARRIERS TO ROUTINE DEPRESCRIBING}

With increased knowledge and successful interventions, comes a stronger impulse for medical professionals 'to treat' every medical illness, whether or not it improves patients' QoL. This can be undertaken by re-evaluation of each drug and discussing with the patient/family the pros and cons of each drug, focussing on the goal of QoL as the patient perceives it. Being easily 
available to medical professionals and combined with patient's pressure and drug companies' propaganda, preventive/curative tools are usually continued in older people including VOCODFLEX, sometimes until death. Globally, the main obstacle to deprescribing is emotional and psychological stress for both practitioners and patients. ${ }^{1}{ }^{33}$ Garfinkel et al ${ }^{1}$ stress that the concept 'deprescribing' may be automatically perceived as a negative approach interfering with our 'good health'. It shatters basic almost 'religious' worldwide beliefs that we all perceive from childhood regarding health-disease and patient-caregiver interactions. The good doctor is supposed to heal but throughout history, the patient-doctor interaction is increasingly perceived as a disease-drug interaction. Most people are disappointed if the interaction with doctors would not eventually transform into some prescription. In the author's experience, when it comes to deprescribing, the patient/family usual response would be "doctor, you recommend stopping this drug, what will you give me instead?"

Traditionally, doctors are perceived as the expert prescribers who wisely choose the right medication/s to treat diseases. The increased rate of overdiagnosis starts a vicious circle; many more apparently healthy people taking many more medications, starting earlier and for longer periods in life. ${ }^{1}$ Numerous other barriers prevent people from asking their physician to reduce the number of medications. Patients fear their doctor's response, fear relapsing, fear being denied the ability to resume medications, and fear abandonment by their physician if they discontinue prescriptions. ${ }^{34-36}$ Doctors have their own barriers, starting with uncertainty over why drugs were prescribed in the first place ${ }^{37}$ they may be concerned about an increased workload, including the time needed to discuss palliative issues such as QoL versus life expectancy. ${ }^{31}{ }^{35}$ Doctors may be afraid not to follow 'guidelines' (not to mention pay-for-performance issues), including all experts' recommendations, even realising that most or all these were not proven in older people, particularly VOCODFLEX. They may fear unfavourable consequences, even if unrelated to deprescribing, afraid of lawsuits and afraid of the patient/family if they dare stop drugs recommended by expert. ${ }^{1}$ Above all, without systematic frameworks to support polypharmacy risk reduction activities, doctors may not feel confident to de-prescribe. ${ }^{38-40}$ Doctors may be frustrated having no EBM RCTs indicating when to stop medications and much uncertainty regarding the effectiveness of strategies to reduce polypharmacy. ${ }^{36} 4041$ Patients and families contribute much to the negative vicious circle of pressures upon doctors. Many disregard doctors' advice to de-prescribe unless the 'experts' approve. Some may even suspect that any attempt of doctors to stop medications is a result of economic incentives, pressure on the doctors from their healthcare organisation management or insurance companies to save money. ${ }^{1}$ Considering the combined effect of these obstacles, most doctors are forced into practising 'defensive medicine'. Consequently, they may be coerced into persuading patients to follow investigation and treatment courses that may not serve that patient's best interests. In a systematic review, Reeve $e t a l^{42}$ concluded:

\footnotetext{
"Understanding the appropriateness of cessation, having a process in place for cessation and a general dislike of medications will facilitate patients' willingness to trial cessation of inappropriate medications. Primary care physicians, family, friends and the media were identified as influences to trialling medication cessation. An effective patient-centred deprescribing process will need to involve patient education on the risks and benefits of ongoing medication use, allaying any fears that patients have about
}

medication cessation, and employing a process that includes support, monitoring and follow-up".

\section{CONCLUSIONS: THE NEW MEDICAL APPROACH FOR OLDER PEOPLE, PARTICULARLY VOCODFLEX}

Reducing the major, iatrogenic 21st century epidemic of inappropriate prescribing should be recognised as a global goal of the highest priority. Our final goal is to achieve better QoL to more older people, for increased periods of time before death. To fulfil this we must revolutionise our basic research and clinical principles while shattering traditional perceptions regarding 'good clinical practice', upon which we have all been educated. The basic demand from each medication is that the benefits would outweigh all possible risks/harm. However, for all drugs the knowledge gap regarding this parameter is increasing in correlation to VOCODFLEX. We have no reliable 'guidelines' for most older people. Nevertheless, they (and we) believe that we are acting based on EBM for their benefit. Doctors' unawareness of their own lack of knowledge represents the highest level of risk for their patients. Doctors are much more cautious if they realise that there are no data proving that prescribing would be better than non-prescribing. This was concluded by Scott et al ${ }^{36}$ who entitled their manuscript: 'First do no harm: a real need to de-prescribe in older patients'. Salazar et al stress that polypharmacy distorts the picture of 'normal' drug effects, 'common' ADE and 'known' interactions. They conclude that we should 'expect the unexpected, think the unthinkable'. ${ }^{43}$ The author suggests a completely new comprehensive perception for providing good medical practice to older people. ${ }^{1}$ It includes new research, education, diagnosis and treatment principles, completely different from 'single disease models' we had been somehow 'fanatically' adopted in the 20th century. The new perception is based on palliative, geriatric and ethical principle, enabling fresh tools for treating VOCODFLEX in general and reducing IMUP in particular.

First, we must internalise the fact that most 20th century research principles of so-called good EBM are not always applicable in most older people (VOCODFLEX). Even inside study and control groups heterogeneity is huge as the impact of VOCODFLEX increases. In deprescribing projects, finding comparable control groups is impossible statistically and may require sample sizes that are greater than the whole subpopulation in question. 'Traditional' RCT tools, statistics and computers are practically impossible. We have not yet arrived at a situation where any computer simulation can show us all possible interactions among multimedications, while factoring in the particular characteristics of a specific older patient. Let us not underestimate the importance of feasibility studies showing that deprescribing results in significantly improved clinical outcomes. Remembering that the natural history of ageing is always downhill, no statistics needed to determine that any significant improvement following excessive drug discontinuation is indeed the result of deprescribing. Researches showing such results are appropriate enough for encouraging doctors to de-prescribe.

We have the advantage of knowing the exact diagnosis-the most significant predictor of IMU is the number of drugs. Polypharmacy is the disease and, therefore, the specific treatment is simple-stopping as many medications as possible. We still have no consensus as for the best way to de-prescribe. In the author's view and that of others, drugs-to-avoid criteria are less useful as stand-alone measures of prescribing. ${ }^{30}{ }^{44}$ Furthermore, the author's view is that stopping one 'inappropriate' medication in a patient with problematic polypharmacy does not go far enough. We have no idea of the whole scope of 
damage caused by the remaining 'appropriate' drugs and there is a paucity of evidence to help us. The author therefore considers it concerning, and perhaps even unethical, to continue prescribing these drugs without review.

The original definition of EBM stresses 'the integration of best research evidence, clinical expertise and patient values'. ${ }^{45}$ While hopelessly searching for reliable best research EBM in older people, we seem to neglect the other two components essential for patient-centred care-clinical judgement and patient/family's preferences. Older people have no time to wait until we solve our confusion having no traditional EBM RCTs or computer solutions. Unfortunately, they suffer from inevitable diseases (God's will if you wish...), and increasingly their major suffering is the result of doctors' induced iatrogenic harm. Defensive medicine trends are turning doctors into medical technicians who automatically, even fanatically, implement inappropriate 'sanctified guidelines' in everyone. It is time we revive forgotten virtues of clinical judgement and simple common sense while ethically incorporating older people/family preferences for their late periods of life.

Finally, the ethical issue; we should realise that routine deprescribing is by no mean ageist. We do not extrapolate from single-disease guidelines in adults to children; similarly, there is no rationale for doing this in older people who, like children, are very vulnerable to ADE. Therefore, individualisation of drug therapy is a must.

It may be concluded that our reluctance to de-prescribe medications unjustifiably increases elders' risk of ADE and IMUP. This situation sharply negates the basic goal of achieving quality of care while complying with our ethical principle, 'primum non nocere'. It even evokes dark thoughts whether we are all, though unconsciously, contributing to the poor care of our most vulnerable patients. This dilemma applies to all doctors as well as other health professionals. It certainly applies to pharmacists who may feel frustrated facing possible inability to slow down the doctor's hand that, traditionally, is the one writing the prescriptions. Globally, as diffusion of responsibility while 'passing the buck' is becoming common practice, who is responsible for stepping on the brakes?

IMUP is a substantial tip of the huge iceberg representing the 'geriatric boom burden'. It may be significantly improved, should we renew our medical perceptions and approach choosing the way suggested here. The author's studies in $\mathrm{NH}$ and community-dwelling elders have proven that carefully planned,

\section{Key messages}

What is already known on this subject?

- Polypharmacy represents a leading 21 st century age-related iatrogenic epidemic.

- No EBM guidelines exist for older people particularly for the most vulnerable subpopulations.

- A variety of obstacles mainly emotional/psychological make routine deprescribing difficult.

What this study adds?

- Focusing on 'drugs to avoid' alone may cause us to disregard the undiagnosed harm imposed by the remaining drugs and interactions.

- Simultaneous massive deprescribing giving high priority to patient/family preferences is highly efficacious and safe. scheduled routine deprescribing is indeed feasible, effective and safe. Extending the ethical, palliative and geriatric principles on which the GPGP is based beyond deprescribing may probably contribute to other age-related health and economic problems.

Facing the vicious cycle of overprescribing fuelled by powerful, for-profit forces, the chance of reversing ingrained psychological-emotional myths regarding drugs seems hopeless. Only an integrated effort of international unprejudiced, nonprofit health professionals, policy makers and consumers, involving the general press and social networks, can win the war against IMUP while enabling better QoL for older people with a triple win-win health-economic gain.

Collaborators IGRIMUP - the International Group for Reducing Inappropriate Medication Use \& Polypharmacy.

Competing interests None declared.

Provenance and peer review Commissioned; externally peer reviewed.

\section{REFERENCES}

1 Garfinkel D, Ilhan B, Bahat G. Routine de-prescribing of chronic medications to combat polypharmacy. Ther Adv Drug Saf 2015;6:212-33.

2 Garfinkel D. Geriatric Boom Catastrophe-A major medical, economic and social nightmare of the 21 st century [abstract]. Proc 6th. Congress Intern Ass Gerontol Geriatr 1997:364.

3 Garfinkel D. The tsunami in 21 st century healthcare: the age-related vicious circle of co-morbidity - multiple symptoms - over-diagnosis - over treatmentpolypharmacy. J Nutr Health Aging 2013;17(Suppl 1):SS24-227-C-1.

4 Rochon PA, Gurwitz JH. Optimising drug treatment for elderly people: the prescribing cascade. BMJ 1997;315:1096-9.

5 Gill SS, Mamdani M, Naglie G, et al. A prescribing cascade involving cholinesterase inhibitors and anticholinergic drugs. Arch Intern Med 2005;165:808-13.

6 de Souto Barreto P, Ferrandez AM, Saliba-Serre B. Are older adults who volunteer to participate in an exercise study fitter and healthier than non volunteers? The participation bias of the study population. J Phys Act Health 2013;10:359-67.

7 Golomb BA, Chan VT, Evans MA, et al. The older the better: are elderly study participants more non-representative? A cross-sectional analysis of clinical trial and observational study samples. BMJ Open 2012;2:e000833.

8 Sugisawa $H$, Kishino $H$, Sugihara $Y$, et al. Characteristics of dropouts and participants in a twelve-year longitudinal research of Japanese elderly. Nihon Koshu Eisei Zasshi 2000;47:337-49. http://www.ncbi.nlm.nih.gov/entrez/query.fcgi? $\mathrm{cmd}=$ Retrieve\&db=PubMed\&dopt=Citation\&list_uids=10835895

9 Hanlon JT, Schmader KE, Ruby CM, et al. Suboptimal prescribing in older inpatients and outpatients. J Am Geriatr Soc 2001;49:200-9.

10 Gnjidic D, Hilmer SN, Blyth FM, et al. Polypharmacy cutoff and outcomes: five or more medicines were used to identify community-dwelling older men at risk of different adverse outcomes. J Clin Epidemiol 2012:65:989-95.

11 Tinetti ME, Bogardus ST Jr, Agostini JV. Potential pitfalls of disease-specific guidelines for patients with multiple conditions. N Engl J Med 2004;351:2870-4.

12 Nightingale G, Hajjar E, Swartz K, et al. Evaluation of a pharmacist-led medication assessment used to identify prevalence of and associations with polypharmacy and potentially inappropriate medication use among ambulatory senior adults with cancer. J Clin Oncol 2015:33:1453-9.

13 Boyd CM, Darer J, Boult C, et al. Clinical practice guidelines and quality of care for older patients with multiple comorbid diseases: implications for pay for performance. JAMA 2005:294:716-24.

14 Angell M. The Truth About Drug Companies: how they deceive us and what to do about it. New York: Random House, 2005.

15 Gotzsche P. Deadly medicines and organised crine. Radcliffe Medical Press, 2013.

16 Healy D. Pharmageddon. University of California Press, 2012.

17 Healy $D$, Cattell $D$. Interface between authorship, industry and science in the domain of therapeutics. Br J Psychiatry 2003;183:22-7.

18 Stavert RR, Lott JP. The bystander effect in medical care. N Eng/ J Med 2013:368:8-9.

19 American Geriatrics Society 2012 Beers Criteria Update Expert Panel. American Geriatrics Society updated Beers Criteria for potentially inappropriate medication use in older adults. J Am Geriatr Soc 2012:60:616-31.

20 O'Mahony D, O'Sullivan D, Byrne S, et al. STOPP/START criteria for potentially inappropriate prescribing in older people: version 2. Age Ageing 2015;44:213-18.

21 Wehling $M$, Burkhardt $H$, Kuhn-Thiel $A$, et al. VALFORTA: a randomised trial to validate the FORTA (Fit fOR The Aged) classification. Age Ageing 2016:45:262-7.

22 Rudolph JL, Salow MJ, Angelini MC, et al. The anticholinergic risk scale and anticholinergic adverse effects in older persons. Arch Intern Med 2008;168:508-13.

23 Hilmer SN, Mager DE, Simonsick EM, et al. Drug burden index score and functional decline in older people. Am J Med 2009;122:1142-9.e1141-1142. 
24 Hanlon JT, Schmader KE. The medication appropriateness index at 20: where it started, where it has been, and where it May be going. Drugs Aging 2013;30:893-900.

25 ARMOR: A Tool to Evaluate Polypharmacy in Elderly Persons|Annals of Long Term Care. http://www.annalsoflongtermcare.com/content/armor-a-tool-evaluate-polypharmacyelderly-persons

26 Moorhouse P, Koller K, Mallery L. End of Life Care in Frailty. Interdiscip Top Gerontol Geriatr 2015:41:151-60.

27 Mallery LH, Ransom T, Steeves B, et al. Evidence-informed guidelines for treating frail older adults with type 2 diabetes: from the Diabetes Care Program of Nova Scotia (DCPNS) and the Palliative and Therapeutic Harmonization (PATH) program. J Am Med Dir Assoc 2013;14:801-8.

28 Moorhouse P, Mallery LH. Palliative and therapeutic harmonization: a model for appropriate decision-making in frail older adults. J Am Geriatr Soc 2012;60:2326-32.

29 Garfinkel D, Zur-Gil S, Ben-Israel J. The war against Polypharmacy. A new, cost Effective Geriatric -Palliative approach for improving drug therapy in disabled older people. Israel Med Assoc J 2007;9:430-4.

30 Garfinkel D, Mangin D. Feasibility study of a systematic approach for discontinuation of multiple medications in older adults. Addressing polypharmacy. Arch Int Med 2010;170:1648-54

31 Scott IA, Hilmer SN, Reeve E, et al. Reducing inappropriate polypharmacy: the process of de-prescribing. JAMA Intern Med 2015;175:827-34.

32 Sengstock D, Zimmerman J, Williams B, et al. Addressing polypharmacy \& improving medication adherence in older adults. Eds: Brie A Williams, Anna Chang, Cyrus Ahalt, Helen Chen, Rebecca Conant, C Seth Landefeld, Christina Ritchie, Michi Yukawa. In: Current diagnosis and treatment: geriatrics 2e. McGraw-Hill Education/Medical, 2014:608.

33 Reeve E, Shakib S, Hendrix I, et al. Review of de-prescribing processes and development of an evidence-based, patient-centred de-prescribing process. $\mathrm{Br} J$ Clin Pharmacol 2014;78:738-47.
34 Linsky A, Simon SR, Bokhour B. Patient perceptions of proactive medication discontinuation. Patient Educ Couns 2015;98:220-5.

35 Reeve E, Turner JP. Patients' perspectives on the brave new word 'de-prescribing'. Int J Pharm Pract 2015;23:90-1.

36 Scott IA, Anderson K, Freeman CR, et al. First do no harm: a real need to de-prescribe in older patients. Med J Aust 2014;201:390-2.

37 Plakiotis C, Bell JS, Jeon Y-H, et al. Deprescribing psychotropic medications in aged care facilities: The potential role of family members. Adv Exp Med Bio 2015;821:29-43

38 Nixon M. Organising medication discontinuation: GP decision making when de-prescribing statins. Denmark: Copenhagen University, 2014.

39 Schuling J, Gebben H, Veehof LJ, et al. Deprescribing medication in very elderly patients with multimorbidity: the view of Dutch GPs. A qualitative study. BMC Fam Pract 2012;13:56.

40 Anderson K, Stowasser D, Freeman C, et al. Prescriber barriers and enablers to minimising potentially inappropriate medications in adults: a systematic review and thematic synthesis. BMJ Open 2014;4:e006544.

41 Ostini R, Hegney D, Jackson C, et al. Knowing how to stop: ceasing prescribing when the medicine is no longer required. J Manag Care Pharm 2012;18:68-72.

42 Reeve E, To J, Hendrix I, et al. Patient Barriers to and Enablers of De-prescribing: a Systematic Review. Drugs Aging 2013;30:793-807.

43 Salazar JA, Poon I, Nair M, et al. linical consequences of polypharmacy in elderly: expect the unexpected, think the unthinkable. Expert Opin Drug Saf 2007;6:695-704

44 Steinman MA, Rosenthal GE, Landefeld CS, et al. Agreement between drugs-to-avoid criteria and expert assessments of problematic prescribing. Arch Intern Med 2009;169:1326-32.

45 Sackett, DL, Rosenberg WMC, Gray JAM, et al. Evidence based medicine: what it is and what it isn't. BMJ 1996;312:71-2. 